\title{
High redshift radio galaxies with the VLT
}

\author{
Huub Röttgering \& George Miley \\ Leiden Observatory, \\ P.O. Box 9513, \\ 2300 RA Leiden, \\ The Netherlands
}

\section{Introduction}

The study of high- $z$ radio galaxies (HZRGs) has matured considerably during the last decade. Large numbers of $z>2$ radio galaxies have been found and have been studied in considerable detail.

During the mid-eighties there were indications from statistical analyses of radio source counts that there might be a cutoff in the space density of powerful radio sources above a redshift of $z \sim 2$ (e.g. Windhorst 1984). However, it has become clear that a substantial population of radio galaxies with $z>2$ does exist. The first radio galaxy discovered with a measured redshift beyond 2 was $4 \mathrm{C} 40.36$ at $z=2.269$ (Chambers et al. 1988). This was soon followed by two radio galaxies at even more extreme distances $(0902+34$ at $z=3.4$, Lilly 1989 and $4 \mathrm{C} 41.17$ at $z=3.8$, Chambers et al. 1990). At the moment the record holder is $6 \mathrm{C} 0140+326$ at $z=4.41$ (Lacy et al. 1996).

Not only has the maximum distance out to which radio galaxies are observed been extended dramatically, but also the number of known galaxies with $z>2$ has grown substantially to well above 100 (e.g. Spinrad 1995, Eales and Rawlings 1996, McCarthy et al. 1996, Röttgering et al. 1996a). In Fig. 1 the redshift distribution of $106 z>2$ galaxies that are known to the authors (mid-1996) is shown. This histogram is of that given by Spinrad (1995) showing the 79 $z>2$ radio galaxies that were known to him in mid-1994. The number of known distant radio galaxies has increased by a third over this two year period.

In this review we will address the importance of the VLT for studies of samples of distant radio galaxies. We shall first briefly discuss the importance of radio galaxies for cosmological studies. Then we shall summarize the various emitting components associated with these objects and comment on the possibilities offered by the VLT for detailed observations of these components and the radio/optical alignment effect. Next, the prospects for detecting and studying galaxy clusters around high redshift radio galaxies are briefly discussed. The case of $1138-262(z=2.15)$ is discussed as an example of a possible proto-galaxy that might be situated in a cluster-type of environment. Finally, we outline some of the steps that should be taken to optimize use of the VLT in this area.

For the most complete review of distant radio galaxies, as per 1993, we refer the reader to McCarthy (1993). 


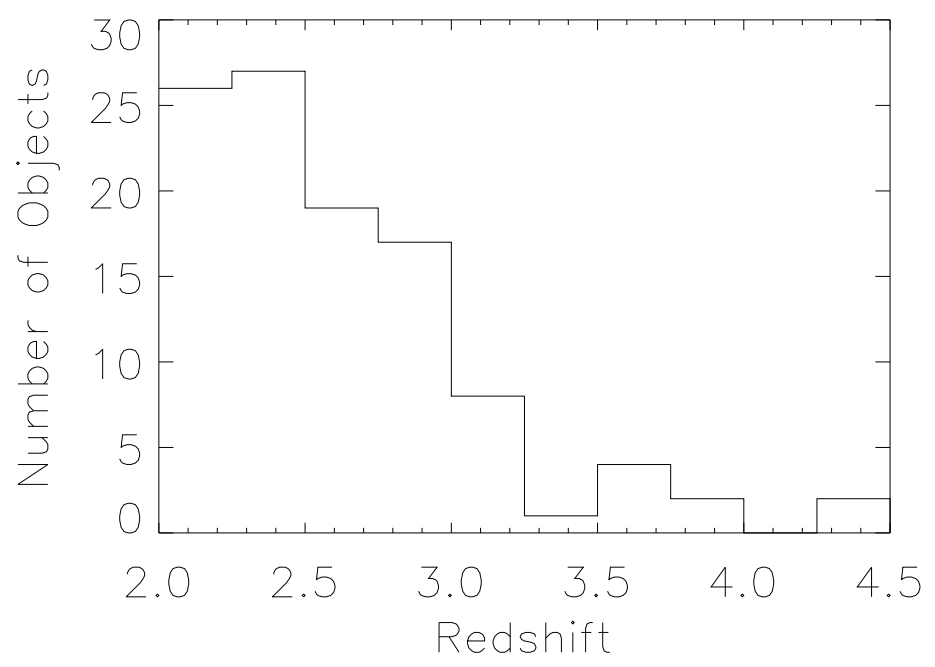

Fig. 1. Redshift distribution of the $z>2$ radio galaxies known to the authors in mid-1996.

\section{Importance}

As a probe of the distant universe, distant radio galaxies offer a wide range of distinct components that can be studied. These include stars, AGN, hot (X-ray emitting) gas, ionized gas, neutral (HI) gas, molecular gas, dust and relativistic plasma. Some components have merely been detected (dust), while others have been extensively studied (ionized gas, neutral (HI) gas, relativistic plasma). It is commonly accepted that these objects contain stars and AGN, but their existence have been inferred by indirect methods. Other components have not yet been detected in the highest redshift objects (molecular gas and hot (X-ray emitting) gas), but it is clear that only modest advances in the performance of instrumentation should allow detection of these components.

Radio galaxies have advantages over quasars as cosmological probes in that at least four of these emitting components can be spatially resolved by ground based telescopes. Furthermore, due to the absence of a bright nucleus, the study of components other than the nucleus itself is less challenging.

One of the aims of studying these distant sources is to better understand processes related to galaxy formation. Possibly the best hint that radio source activity in these distant objects is related to galaxy formation is the strong evolution of the radio luminosity function. The number density of the most powerful steep spectrum radio sources (e.g. $\mathrm{P}_{2.7}=10^{27} \mathrm{~W} \mathrm{~Hz}^{-1} \mathrm{sr}^{-1}$ ) increases by a factor 1000 from $z \sim 0$ up to $z \sim 2-3$ (Dunlop and Peacock 1993). Such strong evolution is a natural consequence in hierarchical models for structure 
formation (Haehnelt and Rees 1993).

Finally, radio galaxies are powerful tracers of galaxy clustering at high redshift. There are a number of observational hints that the highest- $z$ radio galaxies are located in relatively dense environments. We shall discuss this in some detail in section 5 .

\section{Components}

In Table 1 we provide an overview of the mass associated with some fo the components of HZRGs. Most of these estimates are highly model-depended and should therefore treated with caution.

\begin{tabular}{lcl}
\hline \hline Components & Total Mass & Reference \\
& $\mathrm{M}_{\odot}$ & \\
\hline \multirow{3}{*}{ Stars } & $10^{12}$ & $4 \mathrm{C} 41.17, z=3.8$, Chambers et al. (1990) \\
Ionized Gas & $10^{9}$ & $1243+036, z=3.6$, van Ojik et al. (1996a) \\
Neutral (HI) gas & $2 \times 10^{7}$ & $0943-242, z=2.9$, Röttgering et al. (1995) \\
Molecular gas & $<10^{11}$ & Evans et al. (1996); van Ojik et al. (1996b) \\
Dust & $3 \times 10^{8}$ & $4 \mathrm{C} 41.17, z=3.8$, Dunlop et al. (1994) \\
Hot (X-ray emitting) gas & $2 \times 10^{12}$ & 3C356, =1.079, Crawford and Fabian (1993) \\
\hline
\end{tabular}

Table 1. Estimated masses for some of the components associated with distant radio galaxies. A Hubble constant of $H_{0}=50 \mathrm{~km} \mathrm{~s}^{-1} \mathrm{Mpc}^{-1}$ and a density parameter of $\Omega=1$ is used.

Below we will discuss characteristics of the components that can be studied with the VLT and give some recent results indicating what progress can be made using the VLT.

\section{$3.1 \quad$ Stars}

It is clear that characterization of the stellar population in distant galaxies is a prime task for the next generation of optical telescopes. However, the first issue to be addressed is whether there is observational evidence that these systems do indeed contain stars. At low- $z$, powerful radio sources are located in massive ellipticals. By analogy, one would expect this also to be the case at high redshifts. However, optical imaging studies of the morphology of $z>2$ galaxies showed that a large fraction of these objects are clumpy and have its main optical axis aligned with the axis of the radio emission (e.g. McCarthy 1993). This is unlike nearby ellipticals and therefore, before the stellar content of these systems can be properly modelled, it has to be established whether these systems do contain 
stars and, if so, what fraction of the UV/optical/infrared continuum emission is due to stars. We will return to this issue in section 4 . Here we will concentrate on observational evidence that these systems do contain stars.

Until recently the best evidence that these systems do contain a massive population of stars came from infrared imaging. The alignment effect and clumpiness is much less prominent at these relatively long wavelengths and the Hubble Kdiagram shows a continuity from low to high- $z$ and a relatively low dispersion (e.g. McCarthy 1993). All this can be readily explained as related to the existence of a massive population of relatively old stars (for alternative views see Eales and Rawlings 1996).

The best direct evidence for stars comes from recent KECK spectroscopy of a radio galaxy at $z=3.54$ (Dey et al. in prep.). In addition to the usual emission lines (Ly $\alpha, \mathrm{C}$ IV and He II) it showed very strong UV absorption lines. This absorption spectrum very much resembles that of the two nearby Wolf-Rayet galaxies NGC 1741 (Conti et al. 1996) and NGC 4214 (Leitherer et al. 1996) as well as those of the starforming galaxies at $3<z<3.5$ that Steidel et al. (1996) have recently discovered. This similarity clearly indicates that at least one distant radio galaxy contains a massive population of young stars that have been produced during a recent burst of star formation.

The VLT with its proposed optical and infrared instrumentation should allow detailed studies of spectral energy distribution of these systems, including stellar absorption lines. With this information it should be possible to separate the contributions of stellar and non-stellar light to the morphology of the galaxy. This will allow important parameters of these systems to be determined, including stellar content, metallicity, SFR and SF history. The high resolution capabilities of the VLT should allow a number of these parameters to be determined as a function of location within the galaxy. With this information it should be possible to study the distribution of SF over the galaxy and observationally establish the epoch of formation of these galaxies.

\section{$3.2 \quad$ AGN}

Since distant radio galaxies all emit powerful radio emission, it is likely that these objects contain, either now or in the past, active nuclei. During this meeting, Cimatti discussed how observations of scattered light will not only contribute to our knowledge of the scattering medium, but also to that of the nature of the AGN.

It is likely that dust absorbs broad UV emission lines associated with the supposed nucleus in the very centre of the HZRGs. Since dust obscuration is less severe in the infrared, searches for broad emission lines and compact central objects should be carried out in this waveband. In addition, the high resolution imaging of radio loud quasars should give detailed information on the galaxies hosting the quasars. A detailed comparison between these host and those of radio

galaxies should provide important further constrains on models that attempt to unify quasars and radio galaxies. 


\subsection{Ionized gas}

The Ly $\alpha$ emission of distant radio galaxies is often spectacular (e.g. McCarthy 1993). It can be as luminous as $10^{44} \mathrm{erg} \mathrm{s}^{-1}$ and extend up to $100 \mathrm{kpc}\left(\sim 10^{\prime \prime}\right.$ at $z=2.5$ ). These halos could well trace the reservoir of gas from which the galaxies are forming.

Studies of the velocity structure of the gas show that there are two distinct components. The inner region has a relatively high velocity dispersion $(\sim 1000$ $\mathrm{km} \mathrm{s}^{-1}$ ) and there is clear evidence that this is induced by hydrodynamical interactions of the radio jet with the gas. In some sources such interaction is directly observed; in $1243+036(z=3.6)$ the Ly $\alpha$ gas is displaced both spatially and in velocity at the location of a strong bend in the radio jet (van Ojik et al. 1996a). Further evidence for such strong interaction comes from the correlated distortions of the radio and emission line morphologies seen in samples of HZRGs (van Ojik et al. 1996c). The second gas component is the emission line gas outside the main radio source structure, which has a much lower velocity dispersion $(\sim$ $250 \mathrm{~km} \mathrm{~s}^{-1}$ ). In the case of $1243+036$ we have direct evidence that the gas shows ordered motion, possible due to rotation of a protogalactic gas disk at $z=3.6$ out of which the galaxy associated with $1243+036$ is forming. A gravitational origin of the rotation of such a large disk implies a mass of $\sim 10^{12} \sin ^{-2}(i) \mathrm{M}_{\odot}$, where $i$ is the inclination angle of the disk with respect to the plane of the sky.

The distinction between the inner and outer halos has been observed using 4-m telescopes with integration times of order 1 night, about the maximum realistically feasible. An interesting challenge for an 8-m telescope would be to determine the maximum extent of the Ly $\alpha$ halos. The largest sizes measured so far are up to $150 \mathrm{kpc}$. Is this a true cutoff in the distribution or is it possible to trace the Ly $\alpha$ halo out to distances of half a megaparsec?

A very important question concerns the ionisation of the emission line gas. For a number of reasons it is likely that ionisation by a beam from a hidden quasar is playing a role: (i) some HZRGs have a Ly $\alpha$ emitting region that has a cone shaped morphology reminiscent of such a scenario, (ii) the integrated line ratios are well reproduced by nuclear photoionisation models and (iii) there is a tendency for the emission line region to be aligned with the main axis of the radio source (e.g. the optical beam). However, there are a number of problems with this simple picture, the most important being that the outer contours of the Ly $\alpha$ halos are elliptical in shape, and that therefore a significant fraction of halo emission comes from regions perpendicular to the radio axis, ie. not illuminated by the supposed beam. An important constraint on the ionisation mechanisms would be to measure line ratios in these outer regions.

With detailed studies of the morphology, dynamics and ionisation mechanisms of the halos gas, it should be possible to test scenarios for the origin of the gas. It has been suggested that the gas was expelled during a major starburst that the galaxy underwent during its formation. Alternatively, the gas might be indicative of a massive cooling flow that provides a significant fraction of the material from which the galaxy is forming. 


\subsection{Neutral gas}

There are a number of ways to search for and subsequently study neutral gas associated with distant galaxies. One method is to measure the redshifted 21 $\mathrm{cm}$ absorption line against the radio continuum. The only distant radio galaxy for which this has been done is $0902+34(z=3.4)$. Uson et al. (1991) found an absorber in this system with a column density of $4.4 \times 10^{22}$ atoms $\mathrm{cm}^{-2}$, assuming a spin temperature of $10^{4} \mathrm{~K}$. The existence of this absorber was confirmed by Briggs et al. (1993) and de Bruyn et al. (1995). It is likely that more cases will be discovered by the new tunable radio receivers of the Westerbork Radio Telescope (see http://www.nfra.nl/nfra/wsrt_info.html).

A second method is studying the deep narrow troughs that often "disfigure" the Ly $\alpha$ profiles. High-resolution spectra show that, in some cases, these features are too sharp to be explained as separate kinematic components of the emission, but that they are definitely due to absorption by neutral hydrogen along the line of sight. We have analysed deep high resolution spectra for a sample of 18 distant radio galaxies (van Ojik 1995; van Ojik et al. 1996c) and H I absorption features appear widespread in the Ly $\alpha$ profiles. 11 radio galaxies out of the sample of 18 have strong $\left(>10^{18} \mathrm{~cm}^{-2}\right) \mathrm{H} \mathrm{I}$ absorption. Since, in most cases, the Ly $\alpha$ emission is absorbed over the entire spatial extent (up to $50 \mathrm{kpc}$ ), the absorbers must have a covering fraction close to unity. Given the column densities and spatial scales of the absorbing clouds, the typical H I mass of these clouds is $\sim 10^{8} \mathrm{M}_{\odot}$.

On the source with one of the deepest and best defined HI absorption systems (0943-242, $z=2.9$, see also Fig. 4), we have carried out deep high resolution $(1.5 \AA)$ spectroscopy on the C IV and He II line using the AAT telescope. In Fig. 2 we show the resulting spectra. The He II line does not show absorption. This is expected since it is a non-resonant line. The C IV line shows absorption due to the CIV 1548/1551 doublet. We have fitted the line profile with a combination of a Gaussian (for the emission) and two (coupled) Voigt functions (for the absorption). The column density for the absorber is $10^{14.4} \mathrm{~cm}^{-2}$. Combined with the measured column density for the HI absorber $\left(10^{19} \mathrm{~cm}^{2}\right)$, this indicates that the spatially extended absorber is metal enriched. It further shows that such absorption measurement is a good tool to study extended slabs of neutral gas at high redshift. Unfortunately, 0943-242 is one of the few objects for which this kind of work is possible with 4-m class telescopes, since it is among the few that have both strong HI absorption and strong CIV emission.

With the availability of the VLT there is the prospect of conducting detailed studies of such systems. Questions that should be addressed concern the dynamics and morphology of the neutral gas. For example, could the neutral gas be in a rotating system or is it the product of colliding proto-galaxies? Such observations should also address the origin of the gas and its ultimate fate as possible food for forming the stellar populations of the galaxy.

\subsection{Dust}

The existence of dust associated with distant radio galaxies is well established. The most direct method is measuring the dust emission at submillimetre wave- 

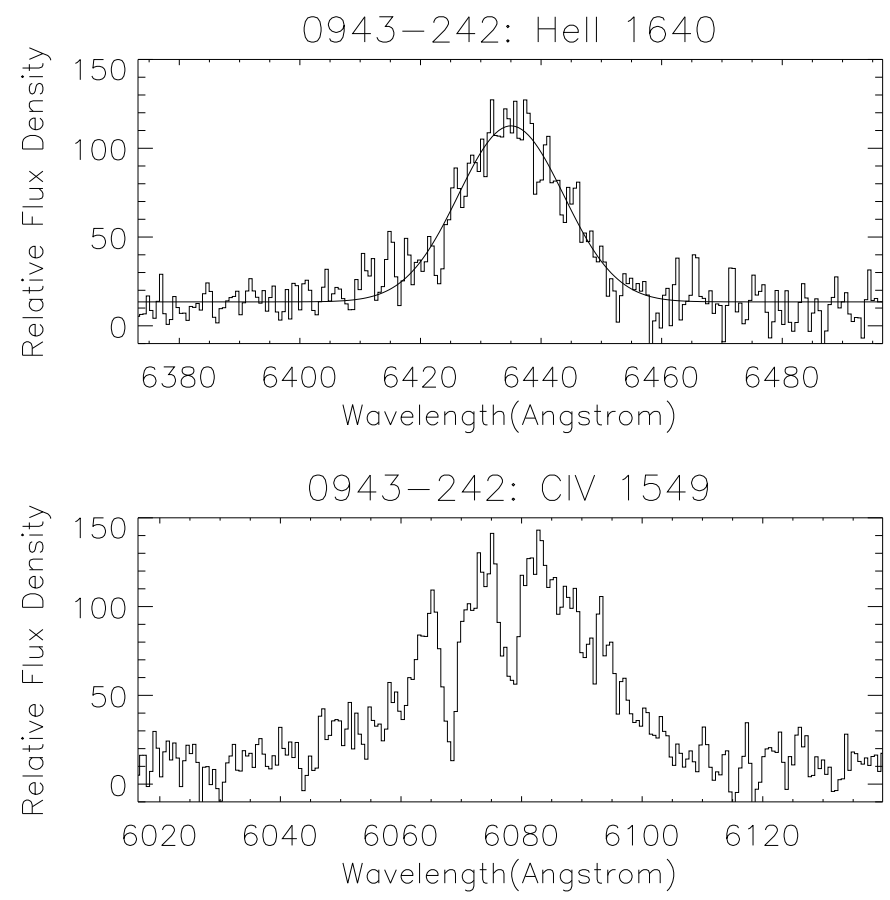

Fig. 2. Parts of the high resolution AAT spectrum (1.5 $\AA)$ of the He II 1640 region (upper) and the CIV 1549 region (lower) of the distant radio galaxy 0943-242 at $z=2.9$ ). The C IV line shows absorption due to the CIV 1548/1551 doublet.

lengths. In Fig. 3 we show the spectral energy distribution of the radio galaxy 4 C41.17 $(z=3.8)$ (from a compilation of Hughes 1996) indicating that this object contains $10^{8} \mathrm{M}_{\odot}$ of dust. Other indirect measurements confirm that HZRGs can indeed contain massive amounts of dust, including (i) the optical/UV polarisation measurements, (ii) the clumpy optical continuum morphologies as compared to those in the infrared and (iii) the $\mathrm{Ly} \alpha / \mathrm{H} \alpha$ emission line ratios.

The amount of dust probably greatly varies from object to object. This is exemplified by two objects (TX0211-122, van Ojik et al. 1994 and MG 1019+0535, Dey et al. 1995) out of an estimated 60 HZRGs that have Ly $\alpha$ very much fainter with respect to the high ionisation lines than in typical high redshift radio galaxies. This suggests that these galaxies are undergoing a vigorous starburst producing a copious amount of dust that attenuates the Ly $\alpha$ emission.

An interesting topic for the VLT will be to compare the spatial distribution of $\mathrm{H} \alpha$ to Ly $\alpha$, thereby estimating the distribution of dust through the whole galaxy. Detailed spectral polarisation measurements with the VLT is another powerful tool for studying the dust distribution. This is discussed by Cimatti in this proceedings. 
Finally, it it is interesting to consider whether the thermal infrared instrument VISIR (VLT Imager and Spectrometer for mid InfraRed) that will be mounted on UT2 is sensitive enough to detect dust at these high redshifts. The $6 \sigma$ RMS in an 8 hour observation is $0.2 \mathrm{mJy}$ for N-band $(8-13 \mu \mathrm{m})$ and $2 \mathrm{mJy}$ for Q-band $(16-24 \mu \mathrm{m})$. We have plotted these two limits in Fig. 3. From this it is clear that dust is only detectable if there is a warm component $(\mathrm{T}>100$ $\mathrm{K})$ comparable in mass to the colder dust. IRAS has established that such warm dust components are a common feature of nearby radio loud AGN (e.g. 3C390.3 Miley et al. 1984). ISO should provide more information. VISIR has the great advantage over ISO in studying such warm dust because of its superb resolution.

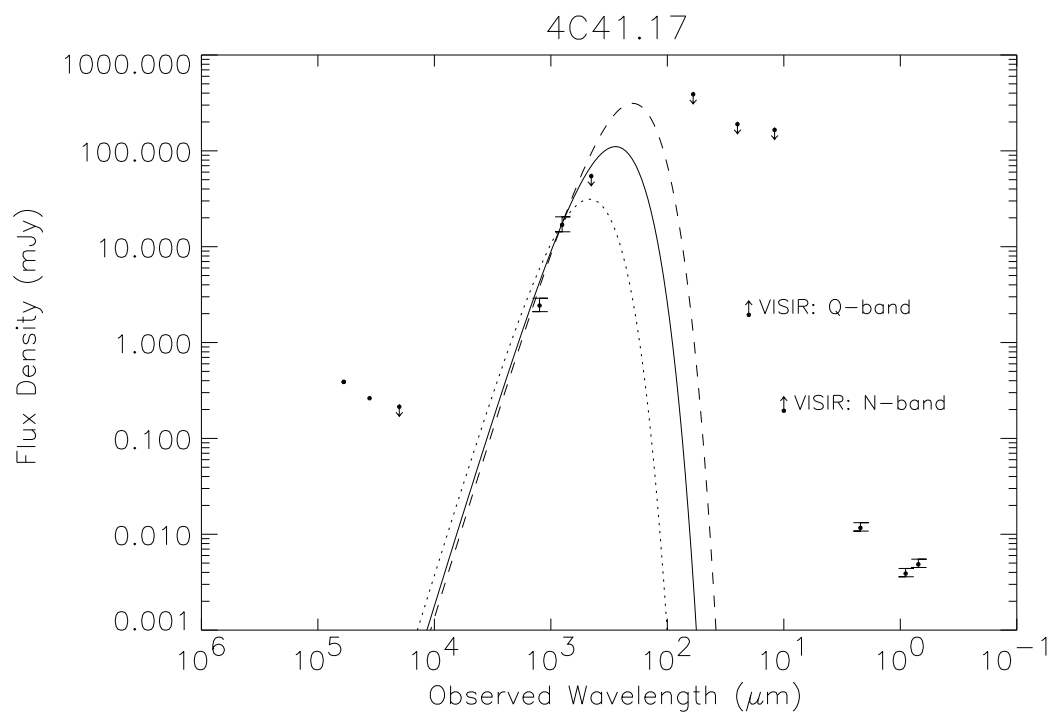

Fig. 3. Spectral energy distributions of the radio galaxy $4 \mathrm{C} 41.17(z=3.8)$; the radio emission is for the core only. The dotted, solid, and dashed lines represent isothermal grey-body emission with an emissivity index $\beta=2$, for dust at temperatures of $30 \mathrm{~K}$, $50 \mathrm{~K}$ and $70 \mathrm{~K}$ respectively. Data are taken from the compilation by Hughes (1996). Also indicated is the $6 \sigma$ RMS in an 8 hour observation for N-band $(8-13 \mu \mathrm{m})$ and Q-band $(16-24 \mu \mathrm{m})$ using VISIR, the mid infrared instrument on the VLT.

\section{The alignment effect}

Several years ago it came as a big shock when it was discovered that, unlike the case for nearby radio galaxies, the optical/IR continuum emission radio emission of $z>0.6$ radio galaxies is roughly aligned with the radio emission (Chambers, Miley and van Breugel 1987; McCarthy et al. 1987). During the last 10 years a 
large number of explanations of this aligned emission have been proposed, the three most promising being scattering of light from a hidden quasar by electrons or dust (Tadhunter et al. 1989; Fabian 1989), star formation stimulated by the radio jet as it propagates outward from the nucleus (Chambers, Miley and van Breugel 1987; McCarthy et al. 1987; De Young 1989; Rees 1989; Begelman and Cioffi 1989) and nebular continuum emission from the emission line gas (Dickson et al. 1995).

The high resolution imaging capabilities of the HST provide excellent opportunities for studying the nature of the interaction of the jet with the host galaxies. At present we are carrying out an imaging survey on a selected sample of $z>2$ radio galaxies. Although the alignment effect is clearly present at the kpc scale, the diversity of structures is enormous. Some of the galaxies have a simple cigar shaped morphologies aligned between the radio lobes (e.g. 0943-242, $z=2.9$, Fig. 4), while others are very complex, showing a number of knots of which some are connected with the radio jet (e.g. $1138-262, z=2.15$, Fig. 5).

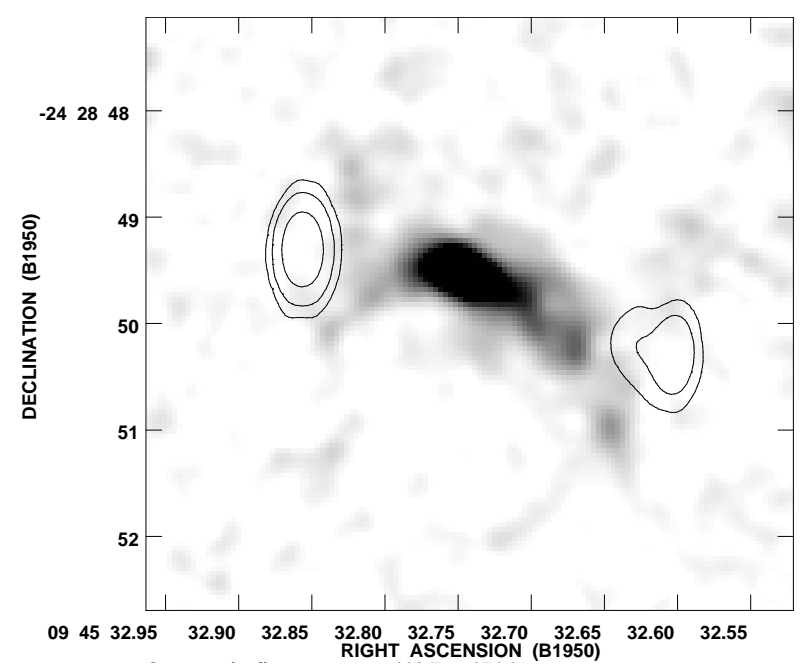

Fig. 4. The grey-scale is the HST image of 0943-242 $(z=2.9)$ through the F702W filter with a total integration time of 5300 sec. The contours show the VLA A-array total intensity radio map at $8.2 \mathrm{GHz}$ with a resolution of $0.25^{\prime \prime}$. The contours are at $(0.2,0.8,4) \mathrm{mJy}$.

At intermediate redshifts we have studied the optical morphologies as observed by HST of a complete sample of $3 \mathrm{CR}$ radio galaxies $(1 \lesssim z \lesssim 1.3)$ and found that they are highly dependent upon their radio properties (Best et al. 1996). There is a clear evolution of the optical structures as the size of the radio source increases: small radio sources consist of many bright knots, tightly aligned along the radio axis, whilst more extended sources contain fewer (generally no 


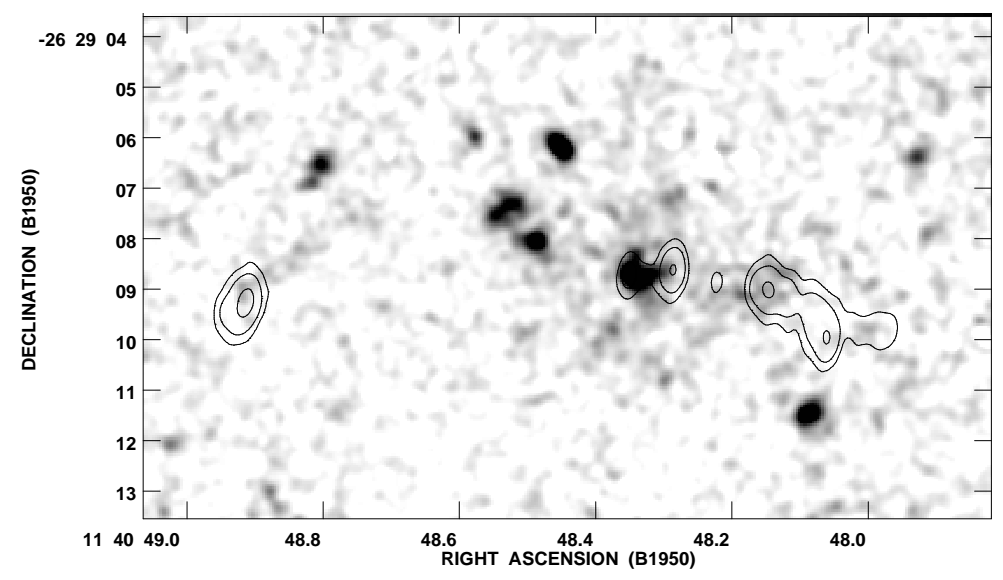

Fig. 5. The grey-scale is the HST image of 1138-262 through the F702W filter with a total integration time of $5300 \mathrm{sec}$. The contours show the VLA A-array total intensity radio map at $8.2 \mathrm{GHz}$ with a resolution of $0.25^{\prime \prime}$. The contours are at $(0.25,1,5) \mathrm{mJy}$.

more than two) bright components and display more diffuse emission.

The morphologies of the intermediate and high- $z$ sources can be explained as a combination of the three explanations as mentioned above. On the basis of the morphologies at the kpc scales most of the alternative models explaining the alignment effect seem no longer tenable including (i) inverse Compton scattering of CMB photons (Daly 1992), (ii) enhancement of radio luminosity by interaction of the jet with an anisotropic parent galaxy (Eales 1992), (iii) alignment of the angular momentum of the nuclear black hole with an anisotropic protogalactic distribution (West 1994) and (iv) gravitational lensing (Le Fèvre et al. 1987).

If it is indeed correct that the UV/optical continuum can be explained as due to a combination of scattered, stellar and nebular continuum light, then the next step is to properly disentangle these three mechanisms. Techniques for this include polarisation, colour information over a large range of wavelengths and narrow band filtering to separate gas from continuum emission. The superior sensitivity of the VLT will be particularly important for such photon limited studies.

\section{$5 \quad$ High redshift clusters}

Detection of clusters and groups of galaxies at high-redshift is important both for constraining cosmological models and for providing unique laboratories for studying a diverse range young and forming galaxies.

High-redshift radio sources are important targets for establishing to what extent clustering exists in the early Universe. At low redshifts $(z \sim 0.1$ to 0.5$)$, luminous steep-spectrum radio sources have long been known to be excellent 
indicators of galaxy clustering (e.g. Miley 1980). It has also been shown that high luminosity radio sources associated with quasars and radio galaxies in the range $0.5<z<1$ are located in rich clusters (e.g. Hill and Lilly 1991).

More recent work clearly indicates that at least some of the radio galaxies at $z \sim 1$ are contained in clusters. The most likely explanation of the X-ray emission detected from some of the $z \sim 13 \mathrm{C}$ radio galaxies (e.g. 3C356, $z=1.079$ ) Crawford and Fabian 1993) is that it originates in a hot halo of X-ray gas associated with a cluster. At this conference Dickinson presented further evidence from Keck spectroscopy and ROSAT X-ray imaging that 3C324 $(z=1.206)$ is located in a cluster (see also Dickinson et al. 1995).

At $z>2$ the existence of clusters around HZRGs has not been established. There are important observational indications, however, that they might be in clusters, including (i) strong Faraday polarisation and rotation of the radio emission of some of the HZRGs indicating dense halos of hot electrons (e.g. Carilli et al. 1996), (ii) an excess of companion galaxies detected along the axes of the radio sources (Röttgering et al. 1996b), (iii) deep K-band imaging showing several red companion galaxies possibly at the same redshift as 4C41.17 $(z=3.8)$ (Graham et al. 1994) and (iv) potential companion galaxies around 4C41.17 discovered through imaging below the Lyman limit (Lacy and Rawlings 1996).

The deep imaging work that can be carried out (and is being carried out) with existing 4-m telescopes provides good candidates for cluster galaxies around HZRGs. To establish the existence of a cluster, the redshifts of these candidate cluster galaxies have to be measured. This will be an important task for the VLT.

\section{$6 \quad 1138-262$ at $z=2.15$ : a young and forming galaxy at the center of cluster?}

Perhaps the ultimate aim of studying distant radio galaxies is to investigate the process of galaxy formation. A number of competing scenarios of galaxy formation have been proposed. Here we mention three classes of models which are currently in vogue and which may be relevant for such studies. First there are cooling flow models in which the galaxy forms during a massive cooling flow. Secondly there are hierarchical models in which dwarf galaxies merge to form the large radio galaxy. Thirdly there are models in which a gas reservoir builds up and undergoes a massive star burst.

This paper is clearly too short to discuss in detail these three different scenarios. Instead what we would like to do here is briefly discuss the remarkable galaxy $1138-262(z=2.15)$ in the light of these three scenarios.

A VLA imaging survey of 34 radio galaxies known to be at $z>2$ has recently been carried out (Carilli et al. 1996) The radio galaxy PKS 1138-262 $(z=2.15$, see Figs. 5 and 6 ) was one of the most extreme objects in the sample having (i) the highest rotation measure (RM) and the largest gradient of RM, (ii) the most distorted radio morphology, and (iii) a spectral index that steepens towards the outer knots. Also the optical properties are very peculiar with (i) a very clumpy 


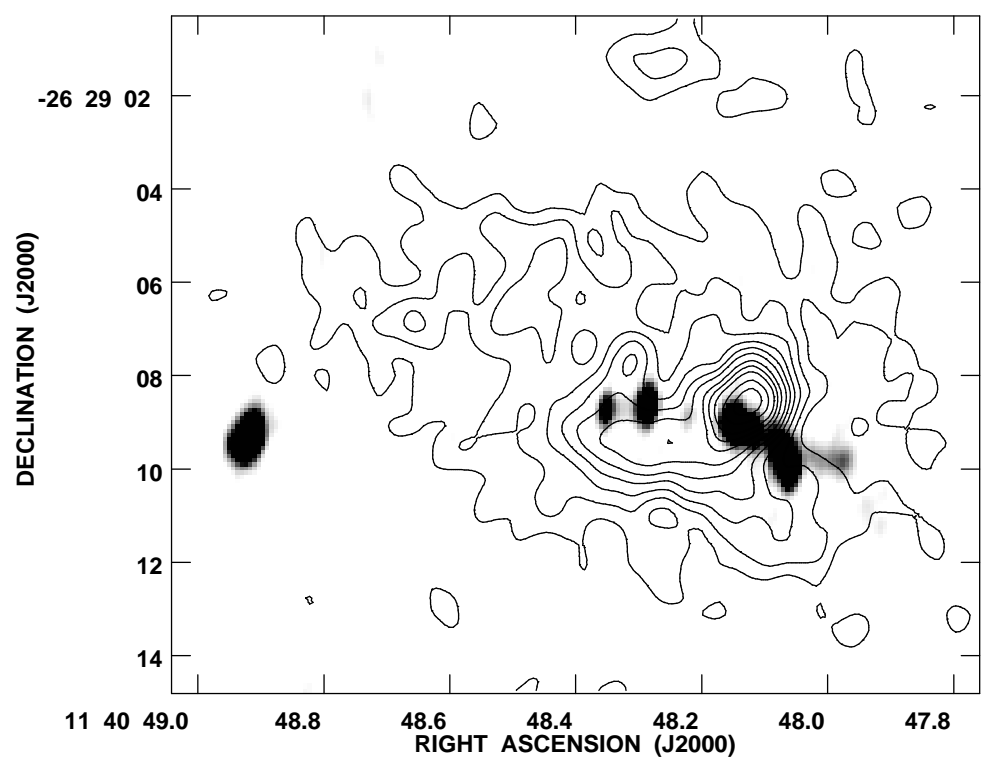

Fig. 6. The contours show an NTT narrow band image of the Ly $\alpha$ emission from 1138-262. The grey-scale represents the VLA A-array total intensity radio map at 8.2 $\mathrm{GHz}$ with a resolution of $0.25^{\prime \prime}$.

continuum emission, as seen by HST, and (ii) a distribution of Ly $\alpha$ emission gas that does not follow the optical continuum (Pentericci et al. 1996).

Drawing the analogy between low redshift radio galaxies and 1138-262 we suggest that this source is at the centre of an extreme cooling-flow, as high as $1000 \mathrm{M}_{\odot} \mathrm{yr}^{-1}$. If this cooling flow could be maintained long enough then it would indeed provide sufficient material from which a large galaxy could be assembled. The extreme clumpiness of this galaxy suggest that that we are witnessing the assembling of this galaxy. Once the subunits have merged, 1138-262 will be a $\mathrm{cD}$ type galaxy at the centre of the cluster.

The suggestion that $1138-262$ is a forming galaxy is possibly premature. It is clear, however, that detailed studies with the VLT of objects like this will allow the hypothesis that such objects are protogalaxies to be tested. Of particular interest will be a detailed determination of the SED of all the individual clumps of these galaxies. What is their stellar content like? Are they indeed SF regions that mainly contain young stars, or are they fairly old dwarf galaxies that happen to be in a cluster around 1138-262? Such measurements are impossible with current 4-m class telescopes and should be carried out with the next generation 
of optical telescopes.

\section{Strategy}

The VLT will be equipped with a broad range of instrumentation, the most important of which are mentioned in Table 2. This table also indicates the major emission components of HZRGs that can studied with each instrument.

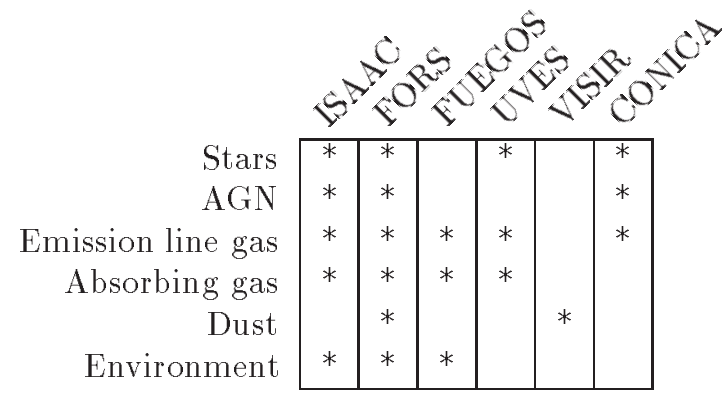

Table 2. An overview of the most important instruments that the VLT will be equipped with, together with the components that can be studied with these instruments.

How can we make optimum use of the VLT for these studies? A first concern is the size and quality of samples of distant radio galaxies that are currently available. Preparatory work should go into a number of projects to obtain: (i) significant numbers of $z>4$ radio galaxies, (ii) complete samples of radio sources that are fully identified and have redshifts, (iii) samples of milli/ $\mu$ Jansky sources and (iv) samples of radio galaxies near bright stars (for adaptive optics/VLTI).

A final word about instrumentation: we believe that with its first set of instrumentation, the VLT is well equipped to carry out studies of the sort that we have mention here. We have a slight concern about the number of narrow and intermediate band filters that will be available. ESO has always had a good filter set, and we hope that it finds ways to provide an adequate set of filters for the VLT instrumentation. Finally, we believe that for studying the environment of distant radio galaxies a wide field infrared imaging capability is essential. We therefore hope that the present plans to build such an instrument will be pursued further.

Acknowledgements. We would like to thank our collaborators, Malcolm Bremer, 
Wil van Breugel, Chris Carilli, Arjun Dey, Dick Hunstead, Jaron Kurk, Laura Pentericci, Pat McCarthy, Rob van Ojik, Hy Spinrad and Paul van der Werf.

\section{References}

Begelman, M. C. and Cioffi, D. F.: 1989, ApJ 345, L21

Best, P., Longair, M. S., and Röttgering, H. J. A.: 1996, MNRAS 280, L9

Briggs, F. H., Sorar, E., and Taramopoulos, A.: 1993, ApJ 415, L99

Carilli, C. L., Röttgering, H., van Ojik, R., Miley, G. K., and van Breugel, W.: 1996, Radio Continuum Imaging of High Redshift Radio Galaxies, APJS: submitted

Chambers, K. C., Miley, G., and van Breugel, W.: 1988, ApJ 327, L47

Chambers, K. C., Miley, G. K., and van Breugel, W.: 1987, Nat 329, 604

Chambers, K. C., Miley, G. K., and van Breugel, W. J. M.: 1990, ApJ 363, 21

Conti, P. S., Leitherer, C., and Vacca, W. D.: 1996, ApJ 461, L87

Crawford, C. and Fabian, A.: 1993, MNRAS 260, L15

Daly, R.: 1992, ApJ 386, L9

de Bruyn, G.: 1995, in M. Bremer, P. van der Werf, H. Röttgering, and C. Carilli (eds.), Cold Gas at High Redshifts, Kluwer, in press

de Young, D. S.: 1989, ApJ 342, L59

Dey, A., Spinrad, H., and Dickinson, M.: 1995, ApJ 440, 515

Dickinson, M., Dey, A., and Spinrad, H.: 1995, in H. Hippelein, K. Meisenheimer, and H. Röser (eds.), Galaxies in the Young Universe. Springer-Verlag, p. 164

Dickson, R., Tadhunter, C., Shaw, M., Clarck, N., and Morganti, R.: 1995, MN$R A S \mathbf{2 7 3}, \mathrm{L} 29$

Dunlop, J. S., Hughes, D. H., Rawlings, S., Eales, S. A., and Ward, M. J.: 1994, Nat 370, 347

Dunlop, J. S. and Peacock, J.: 1993, MNRAS 263, 936

Eales, S. A. and Rawlings, S.: 1992, in Infrared Spectroscopy: future observational directions, preprint

Eales, S. A. and Rawlings, S.: 1996, ApJ 460, 68

Evans, A. S., Sanders, D. B., Mazzarella, J. M., Solomon, P. M., Downes, D., Kramer, C., and Radford, S.: 1996, ApJ 457, 658

Fabian, A. C.: 1989, MNRAS 238, 41P

Graham, J. R., Matthews, K., Soifer, B. T., Nelson, J. E., Harrison, W., Jernigan, J. G., Lin, S., Neugebauer, G., Smith, G., and Ziomkowski, C.: 1994, ApJ 420, L5

Haehnelt, M. G. and Rees, M. J.: 1993, MNRAS 263, 168

Hill, G. and Lilly, S.: 1991, ApJ 367, 1

Hughes, D.: 1996, in M. Bremer, P. van der Werf, H. Röttgering, and C. Carilli (eds.), Cold Gas at High Redshifts, Kluwer, in press

Lacy, M. and Rawlings, S.: 1996, MNRAS 280, 888

Lacy, M., Rawlings, S., Wold, M., Bunker, A., Blundell, K. M., Eales, S. A., and Lilje, P. B.: 1996, in C. Parma (ed.), IAU Symposium No. 175: Extragalactic Radio Sources, Kluwer, in press 
Le Fèvre, O., Hammer, F., Nottale, L., and Mathez, G.: 1987, Nat 326, 268

Leitherer, C., Vacca, W. D., Conti, P. S., Filippenko, A. V., Robert, C., and Sargent, W. L. W.: 1996, ApJ 465, 717

Lilly, S. J.: 1989, ApJ 340, 77

McCarthy, P., van Breugel, W., Spinrad, H., and Djorgovski, S.: 1987, ApJ 321, L29

McCarthy, P. J.: 1993, ARA\&A 31, 639

McCarthy, P. J., Kapahi, V. K., van Breugel, W., Persson, S. E., Athrea, R., and Subramhanya, C. R.: 1996, APJS: submitted

Miley, G. K.: 1980, ARA\&A 18, 165

Miller, L.: 1984, in R. Fanti, K. Kellerman, and G. Setti (eds.), VLBI and compact radio sources, p. 189, Dordrecht:Reidel

Pentericci, L., Röttgering, H., and Miley, G.: 1996, 1138-262: a young and forming galaxy at the center of a cluster, AA, in prep.

Rees, M. J.: 1989, MNRAS 239, 1P

Röttgering, H., Hunstead, R., Miley, G. K., van Ojik, R., and Wieringa, M. H.: 1995, MNRAS 277, 389

Röttgering, H., van Ojik, R., Miley, G., Chambers, K., van Breugel, W., and de Koff, S.: 1996a, Spectroscopy of Ultra-Steep Spectrum Radio Sources: A sample of $z>2$ Radio Galaxies, A \& A: in press

Röttgering, H. J. A., West, M., Miley, G., and Chambers, K.: 1996b, A\&\&A 307, 376

Spinrad, H.: 1995, in H. Hippelein, K. Meisenheimer, and H. Röser (eds.), Galaxies in the Young Universe. Springer-Verlag, p. 95

Steidel, C. C., Giavalisco, M., Pettini, M., Dickinson, M., and Adelberger, K. L.: 1996, ApJ 462, L17

Tadhunter, C. N., Fosbury, R. A. E., Binette, L., Danziger, I. J., and Robinson, A.: 1987, Nature 325, 504

Uson, J., Bagri, D. S., and Cornwell, D. S.: 1991, Phys. Rev. Letter 67, 3328

van Ojik, R.: 1995, Ph.D. thesis, University of Leiden

van Ojik, R., Röttgering, H., Carilli, C., Miley, G., and Bremer, M.: 1996a, A radio galaxy at $z=3.6$ in a giant rotating Lyman $\alpha$ halo, A\&A: in press

van Ojik, R., Röttgering, H., Miley, G., Bremer, M., Macchetto, F., and Chambers, K.: 1994, A\&̈A 289, 54

van Ojik, R., Röttgering, H., van der Werf, P., Miley, G., Carilli, C., Isaac, K., Lacy, M., Jenness, T., Sleath, J., Visser, A., and Wink, J.: 1996b, A search for molecular gas in high redshift radio galaxies, A\&A: in press

van Ojik, R., Röttgering, H. J. A., Miley, G. K., and Hunstead, R.: 1996c, The Gaseous Environment of Radio Galaxies in the Early Universe: Kinematics of the Lyman a Emission and Spatially Resolved HI Absorption, A\&A: in press

West, M. J.: 1994, MNRAS 268, 79

Windhorst, R.: 1984, Ph.D. thesis, University of Leiden 